Techniques \& Culture

Revue semestrielle d'anthropologie des techniques

43-44| 2004

Mythes. L'origine des manières de faire

\title{
Le récit des deux tisseuses
}

\section{Véronique Pardo}

\section{OpenEdition}

Journals

Édition électronique

URL : https://journals.openedition.org/tc/1257

DOI : $10.4000 /$ tc. 1257

ISSN : 1952-420X

Éditeur

Éditions de l'EHESS

Édition imprimée

Date de publication : 1 décembre 2004

ISSN : 0248-6016

Référence électronique

Véronique Pardo, «Le récit des deux tisseuses», Techniques \& Culture [En ligne], 43-44 | 2004, mis en ligne le 17 mars 2006, consulté le 29 septembre 2022. URL : http://journals.openedition.org/tc/1257 ; DOI : https://doi.org/10.4000/tc.1257

Ce document a été généré automatiquement le 29 septembre 2022

Tous droits réservés 


\title{
Le récit des deux tisseuses
}

\author{
Véronique Pardo
}

1 Dans le cadre de la réflexion proposée autour des relations entre mythes et techniques, mon propos est de comprendre l'origine d'un procédé de tissage au travers d'un récit relevé à Douiret -village berbérophone du sud-est tunisien ${ }^{1}$ - qui expose un savoirfaire, en l'occurrence tisser, mais aussi une manière d'être et une manière de faire constitutive de l'«entre-nous $»^{2}$. L'étude menée ${ }^{3}$ a mis en évidence que le tissage construit une des institutions principales de la société douiri, le mariage. La particularité des techniques -à la fois gestes matériels et phénomènes sociaux (Cresswell 2001: 597)- permet d'accéder à la société par l'étude des gestes et des savoir-faire. Parallèlement, par ses récits et ses rituels, la société éclaire les gestes. Cette approche illustre le caractère heuristique de la technologie culturelle (Bonte 1999: 22; Warnier 1999: 26-59) qui, par la nature même de son objet, intègre une perception holiste.

2 À Douiret, les tissus et le savoir-faire de la femme en matière de tissage constituent le «trousseau de la mariée ». Le tissage recouvre une relation sociale qui met en jeu la valeur de l'honneur véhiculé par les objets tissés. Seules les femmes tissent, et les tissus sont des biens féminins grâce auxquels elles participent aux échanges, notamment au plus important, celui qui crée, qui inaugure leur propre foyer. Ces objets sont d'ailleurs aujourd'hui majoritairement destinés à l'habitation (couvertures, tapis avec ou sans motifs) $)^{4}$ et de moins en moins à l'habillement ${ }^{5}$, ce qui était encore le cas il y a une vingtaine d'années.

3 Un récit décrit la particularité de la manière de tisser à Douiret, ainsi qu'un impératif rituel ouvrant l'ourdissage. Il permet de comprendre la société : les limites du terroir, les spécificités du tissage, la conception de la personne comme être relationnel incarné dans le récit par "les deux tisseuses ", techniquement mis en gestes par le tissage, rituellement par les noces et en mots par cette histoire. Dès leur plus jeune âge, les jeunes enfants en connaissent une version ludique, sous forme de berceuse; dans le récit, cette berceuse est chantée par la femme à l'attention de son fils pour l'avertir de la présence de « l'autre tisseuse » qui travaille à ses côtés. Voici la version recueillie. 
Une femme, le soir, tissait en chantant des berceuses pour son bébé. Elle trouvait que ses kilim $^{6}$ [tapis] avançaient exceptionnellement vite et elle se demandait si quelqu'un ne tissait pas pour elle. Un jour dans la demi-pénombre des lampes à pétrole, en entrant, elle vit un « être » aux yeux rouges et aux cheveux rouges qui tissait sur son métier. Elle voyait souvent cette tamzat [génie féminin] assise à côté d'elle, qui tissait, et ses tapis avançaient. Elle en parla à son fils aîné qui ne la crut pas car chaque fois qu'il entrait, il ne voyait que sa mère. Elle inventa donc une petite chanson en chelha et ils s'entendirent sur le fait que lorsqu'elle chanterait, il essaierait d'apercevoir, de loin, le fameux « génie ». Ce qu'ils firent, et le fils dut se rendre à l'évidence.

La même femme distribuait de la bsissa [nourriture rituelle à base de farine de céréales et de légumineuses] en différents lieux dans la petite vallée jusqu'à Sidi Bouana. Un jour, une fois la distribution achevée, il en restait un peu dans une assiette, juste de quoi nourrir son fils et elle. Elle posa l'assiette devant la porte; de l'intérieur elle voyait la bsissa briller comme de l'or; elle est allée voir, c'était simplement la bsissa. Le lendemain matin, le contenu de l'assiette était devenu de l'or.

Après une présentation et une analyse formelle du texte, afin de situer et d'expliciter les nombreuses références aux valeurs de la société ainsi qu'à son environnement, l'ordre de l'énonciation sera respecté ${ }^{7}$ pour présenter d'abord l'origine de la technique de tissage puis le rituel ouvrant l'ourdissage.

$\mathrm{Au}$ fil du récit

5 Ce récit prend pour cadre l'ancien village de Douiret (un ksar, village fortifié de montagne), et il nous présente la limite nord du terroir ${ }^{8}$, le sanctuaire de Sidi Bouana. Les Douiri qui me l'ont raconté précisent toujours que l'action se déroule dans cet ancien village, en situant même très précisément la maison des protagonistes dans le quartier d'un des lignages importants, celui des Ouled taleb. Aujourd'hui, le village de Douiret est composé de deux ensembles : l'ancien village sur et dans la montagne puisque des habitations troglodytiques y étaient creusées horizontalement, et le nouveau village, construit il y a moins d'une trentaine d'années, en contrebas, dans la vallée encaissée. Le déménagement de l'ancien au nouveau village a été progressif et la dernière famille s'est installée dans la vallée en 1998. Dire et penser le village correspond souvent au village fortifié, le ksar, le nouveau village étant perçu comme «l'endroit où l'on dort». Le terroir est délimité par plusieurs sanctuaires, des tombeaux de saints (wouli) dont Sidi Bouana est l'un des plus importants : c'est le lieu où, sept jours après les noces, les nouveaux mariés partent en procession renforcer leur union par la demande de la baraka du saint, ce qui officialise le nouveau foyer et l'ancre dans la communauté.

Plusieurs personnages interviennent dans ce récit et il convient de les présenter en analysant les relations qui les unissent.

7 Il y a d'abord la première femme, mère d'au moins deux enfants puisqu'il est fait allusion à un bébé pour qui elle chante des berceuses, et à son fils aîné. Elle doit donc avoir, ou avoir eu, un mari qui n'apparaît pas dans le récit mais qui appartient à la grande famille des Ouled taleb puisque la résidence est virilocale.

8 Le deuxième être féminin, la tamzat ${ }^{9}$, est un génie. L'influence de ce type de génie sur la destinée est le plus souvent connotée négativement, mais ici son intervention est bénéfique. L'affirmation selon laquelle la femme est tamzat découle du portrait tracé dans le récit: une femme aux yeux rouges et aux cheveux rouges. À Douiret, cette description, dans une conversation, suffit à indiquer que l'on change de registre, que 
l'on aborde un récit, ou que l'on fait une plaisanterie. Dire d'une femme que c'est une tamzat résume un ensemble de défauts dont un caractère peu affable, très autoritaire, et parfois des traits physiques ou vestimentaires excentriques par rapport aux normes du village.

9 La femme qui a reçu la visite de la tamzat n'est pas effrayée. Son fils aîné ne peut la voir qu'un instant, par la porte entrouverte, grâce au subterfuge que sa mère et lui mettent au point, qui consiste en une berceuse adressée en apparence au bébé mais qui est en fait un code entre eux deux. Ils doivent donc passer par le cadet pour s'entendre; on dit souvent que le dernier né d'une longue fratrie est plus choyé, car une mère a pour lui des préférences analogues à celles qui sont dues au fils aîné. Une mère n'a pas intérêt à ce que sa parole soit mise en doute par son fils aîné, qui pourrait, une fois marié, donner raison à son épouse contre elle. Ici, le fils reconnaît que sa mère a raison en apercevant la tamzat qui tisse. Il faut noter que lorsque le récit m'a été raconté, on m'a précisé que la berceuse était en chelha. Or cette berceuse, code entre la mère et son fils, parle de la femme aux cheveux rouges à son insu; cela suppose donc que cette dernière ne comprend pas le chelha, et n'est donc pas douiri. Je reviendrai sur ce fait dans l'analyse de la technique de tissage, ainsi présentée de manière allégorique.

La mère de famille a l'habitude de faire des offrandes de bsissa dans la vallée ${ }^{10}$. La composition de la bsissa est complexe : du blé, de l'orge, des lentilles, des pois-chiches et des peaux séchées d'oranges sont grillés à sec dans un récipient en métal posé sur un feu de bois dans la cour de la maison ${ }^{11}$; ces ingrédients sont ensuite moulus ${ }^{12}$. La farine ainsi obtenue est la base de la bsissa. Son accommodement a lieu juste avant la consommation : les femmes mouillent la farine d'un peu d'eau ou d'huile d'olive, voire des deux. Selon le goût souhaité, on ajoutera ou non du sucre. On obtient une pâte sèche, friable et granuleuse (dorée ou marron) qui sera pétrie en petits pâtons par le consommateur au moment de la porter à sa bouche. À Douiret, la bsissa est présente à tous les moments importants: les femmes la partagent avant d'ourdir un métier à tisser; les noces s'ouvrent par un «repas » de bsissa dans la maison des parents de la future épouse. Le dépôt de cette nourriture sur des pierres ou à des endroits particuliers (arbre, source...) doit attirer la baraka sur le donateur et les lieux où l'offrande est effectuée. La femme du récit en distribue jusqu'à Sidi Bouana, un des sanctuaires situés au nord sur le plateau à l'arrière du village. Le récit, en le citant, délimite géographiquement le cadre de l'histoire et ancre localement les valeurs qu'il met en scène.

11 Lié à la bsissa dont il est issu, l'or apparaît dans le récit après une nuit (« le lendemain matin...») pendant laquelle s'opère la transformation. Au village, seuls les bijoux des femmes, offerts lors du mariage, sont en or. Il s'agit d'un don du futur époux à sa future épouse, et même si ce don intervient au cours de la semaine qui précède les noces, il n'appartiendra à l'épousée que le matin qui suit la nuit de noces. L'or transite dans le cadre traditionnel à cette unique occasion. On ne peut pas ourdir un métier sans manger de la bsissa, on ne peut commencer une noce sans partager la bsissa, et on ne peut consommer le mariage sans don du précieux métal de l'homme à la femme. On ne peut rien faire sans passer de la bsissa à l'or. Dans la famille que présente le récit, apparaît comme une incomplétude : il n'y a pas d'homme, à part le fils, et on ignore si son père est vivant ou mort. D'ordinaire, la femme distribue de la bsissa dans la vallée comme il est fait lors des noces dans la maison de la mariée; le reste de bsissa est transformé en cet or qui est la compensation matrimoniale traditionnelle au sein d'un 
ensemble codifié de prestations. La métaphore du mariage est ici repérable dans la nourriture et dans le métal précieux, mais également dans le tissage.

En effet, la femme a reçu deux signes apparemment différents mais énoncés de manière à ce que l'un découle de l'autre : l'aide pour le tissage puis la bsissa transformée en or. Ces deux événements du récit, toujours ordonnés de la même façon, peuvent s'interpréter ainsi : le tissage quotidien permet la transformation de la bsissa en or. En première lecture, nous avons trois variables; Toutefois la bsissa est changée en or, les deux éléments deviennent donc homologues et nous pouvons réduire l'ensemble à deux variables. Le tissage amène à l'or : à l'occasion des noces, la future épouse, aidée de sa parenté, constitue un trousseau de pièces tissées. Leur facture (finesse et organisation des motifs, choix des couleurs) permet à la future belle-famille de juger du niveau de raffinement et de la place de la famille de la jeune fille dans la société; cela permet aussi d'évaluer la valeur de la future mariée. Car à Douiret, " être bonne à marier » c'est avant tout maitriser la technique, savoir associer les couleurs et apprécier la qualité de la laine, c'est-à-dire être une bonne tisseuse.

Tisser à deux, être deux, être douiri

Le métier à tisser en usage à Douiret et dans la région est un métier vertical dit à hautes lisses sur cadre. En tant qu'ensemble en action, c'est-à-dire l'ensemble des pièces qui le constituent, il est nommé $a z t a^{13}$ en chelha. On le démonte une fois la pièce terminée. Faire l'ourdissage, c'est refaire, créer le métier à tisser à chaque fois puisque ce dernier n'existe nommément que lorsque tous ses éléments sont liés, enserrés les uns aux autres, et que la chaîne verticale est attachée au mur intérieur de la maison par la barre des lisses.

14 Le métier à tisser est toujours installé dans la maison, jamais en plein air; il fait généralement face à la porte : l'endroit du tapis est orienté vers l'extérieur, l'envers vers l'intérieur. Cette orientation apparait d'ailleurs dans la façon de nommer les deux faces du tissu: dire l'envers et l'endroit, c'est dire l'intérieur et l'extérieur, tant en termes linguistiques qu'en termes de représentations. La chaîne se dresse donc verticalement en séparant deux espaces : le plus large, vers l'extérieur, vers la porte, et celui où s'installe la tisseuse, très étroit, entre le mur intérieur et la chaîne. Quand les femmes tissent à deux, elles s'installent dans cet espace intérieur, côte à côte, chacune devant une moitié de chaîne. Par sa position, le métier à tisser distingue deux espaces : un espace strictement féminin, celui d'où l'on voit l'envers du tissage, le dedans, entre le mur intérieur et la chaîne, et un espace que l'on peut qualifier de masculin d'où l'on ne peut voir que l'endroit du tissage, le dehors, l'extérieur, situé du côté de la porte. Les poteaux verticaux qui portent les ensouples sont attachés au mur extérieur, la barre des lisses au mur intérieur.

15 Le récit souligne un tissage à quatre mains : «Elle voyait souvent cette femme assise à côté d'elle, qui tissait et ses tapis avançaient». La mère, tisseuse, est assistée sans l'avoir demandé. La tamzat tisse pour elle et, plus important, à côté d'elle; elles partagent donc la chaîne. Ainsi est décrite la spécificité du tissage à Douiret et son origine : les fils de trame ne sont pas passés sur toute la largeur de la chaîne en une seule fois, mais par plusieurs va-et-vient partiels, successifs, et répétés jusqu'à ce qu'ils soient conduits sur toute la largeur. Cette technique est celle de la tapisserie, normalement employée pour créer des motifs ou pour changer de couleur en cours de bande. Or, dans le village, la technique de la duite partielle ou technique du kilim (au 
sens générique et non local du terme) est également utilisée dans les bandes monochromes et ne sert pas uniquement à un changement de couleur ou de fil.

Le tableau suivant décrit l'opération de tissage au sens strict, d'après mes observations de tisseuses travaillant en solitaire ou à deux. Nous n'incluons pas toutes les phases de la création d'un tissu (tissage des cordelines, arrêt du tissu, ajout...) mais seulement des traits spécifiques de la technique :

\begin{tabular}{|c|c|c|}
\hline Opérations & Détails gestuels & Outils \\
\hline $\begin{array}{l}\text { 1- Passer les fils de trame. } \\
\text { D'une main, la tisseuse introduit un fil d'un côté de } \\
\text { la chaîne en poussant avec le bout des doigts. De } \\
\text { l'autre main, elle tire le fil vers l'autre côté de la } \\
\text { chaîne sur une partie seulement de la largeur de } \\
\text { cette dernière (duite partielle). }\end{array}$ & $\begin{array}{l}\text { L'ensemble de ces gestes est } \\
\text { reproduit plusieurs fois à } \\
\text { chaque inversement de pas. } \\
\text { Quand deux tisseuses tissent } \\
\text { ensemble, l'ampleur des } \\
\text { gestes est réduite à une } \\
\text { moitié verticale de la chaîne. }\end{array}$ & Mains \\
\hline $\begin{array}{l}\text { 2- Écraser les fils. } \\
\text { À l'aide d'un bâtonnet effilé et dur, la tisseuse passe } \\
\text { entre chaque fil de chaîne pour aplanir les fils de } \\
\text { trame afin d'ajuster toutes les duites. }\end{array}$ & $\begin{array}{l}\text { Geste rapide et vif reproduit } \\
\text { sur toute la largeur ou la } \\
\text { moitié seulement de la } \\
\text { chaîne. }\end{array}$ & $\begin{array}{ll}\text { Ligule } & \text { de } \\
\text { palme } & \text { de } \\
\text { palmier } & \\
\text { dattier. } & \\
\text { Une par } \\
\text { tisseuse }\end{array}$ \\
\hline $\begin{array}{l}\text { 3- Tasser les fils } \\
\text { Les duites précédemment apla-nies et formant une } \\
\text { « seule » bande sont tassées afin d'être très serrées }\end{array}$ & $\begin{array}{l}\text { Geste rapide et appuyé } \\
\text { repro-duit sur toute la } \\
\text { largeur ou la moitié } \\
\text { seulement de la chaîne. }\end{array}$ & $\begin{array}{l}\text { Peigne en } \\
\text { métal } \\
\text { Un par } \\
\text { tisseuse }\end{array}$ \\
\hline
\end{tabular}

Dans ce type de tissage, la chaîne est toujours "partagée ». Chaque duite est arrêtée à un endroit différent dans la hauteur afin d'éviter une marque visible (césure) une fois le produit achevé. Il y a donc une asymétrie dans ce travail de duites partielles, tant par rapport à la largeur de la chaîne qu'à l'ensemble du tapis. La duite partielle est une technique de tissage à deux: réellement quand deux femmes tissent ensemble ou seulement techniquement quand la tisseuse est seule et qu'elle " partage » sa chaîne de manière différente et dissymétrique à chaque passage de fils de trame. Ce tissage à deux -d'un point de vue technologique- illustre ce qu'est le tissage dans la société : une relation masculin/féminin, horizontal/vertical, intérieur/extérieur que l'on retrouve dans les rituels. La dissymétrie de la technique s'oppose à la symétrie totale des motifs tissés, margoum, et de ces motifs dans un ensemble donné (kilim, farachiya). Quand deux femmes tissent ensemble, elles intercalent leurs duites d'un rang sur l'autre afin d'éviter la césure au milieu de la pièce tissée. La dissymétrie inhérente à la technique est alors compensée par la complémentarité des deux tisseuses.

Deux femmes qui tissent ensemble et en même temps sont en général issues d'une même famille: mère/fille, sœur/sœur, nièce/tante maternelle ou paternelle; lorsque l'entente est cordiale, elles peuvent être belles-sœurs ou encore amies très proches et de préférence du même lignage. Il faut donc qu'elles soient déjà intimes. Or, dans le 
texte analysé, la maîtresse de maison ne connaît pas la femme aux cheveux rouges qui l'aide à tisser; elle la surprend un jour dans la pénombre. Elles tissent ensemble mais la tamzat n'est pas toujours visible ${ }^{14}$. Quand la femme est seule devant son ouvrage, elles sont quand même deux, et c'est là toute la subtilité de la technique de la duite partielle qui correspond toujours à "un tissage à deux ». La femme semble s'être habituée à cette "compagnie » mais elle reste intriguée. Elle invente un subterfuge pour mettre son fils au courant et expliquer la raison de l'avancement rapide de ses ouvrages. La langue chelha permet au fils, et par son intermédiaire à toute la société, d'accéder à la connaissance de l'existence de «la deuxième tisseuse », c'est-à-dire de la technique de la duite partielle, présentée comme plus rapide et produisant un tissu de qualité. L'accès à la reconnaissance du caractère particulier de cette technique se fait grâce à l'existence et à la connaissance exclusive de la langue du groupe qui permet de parler l'entre-nous. La tamzat, qui ne comprend pas le chelha, est doublement autre du fait de sa nature (génie) et de son non-entendement de la langue. Par ce récit, nous retrouvons la relation toujours présente dans ce village entre ce qui est intérieur (langue chelha, village, maison, femme, envers du tissage...) et ce qui est extérieur (langue arabe, jardins, homme, endroit du tissage...) ici presque inversé par le mythe mais qui garde toute sa cohérence ${ }^{15}$.

19 L'extérieur est donc présent dans l'origine de cette technique qui singularise pourtant le tissage de cette région. Ces éléments, que nous ne pouvons développer plus longuement ici, montrent que la lecture de l'appartenance au groupe au travers des faits techniques ${ }^{16}$ participe totalement de la compréhension de ce récit et de la société de Douiret. La technique de tissage y exprime une valeur sociale fondamentale que l'on peut appeler "l'être relationnel ». À Douiret en effet, être adulte c'est être mariée ${ }^{17}$, c'est-à-dire être deux -un couple. Une personne ne se comprend que dans cette relation, elle est constituée "d'un et un", aussi bien dans sa définition sociale (une "personne» est forcément mariée) que dans ses composantes internes (une âme humide et un souffle chaud). Tisser selon cette technique rencontre à deux niveaux la conception douiri de la personne. D'abord l'acte de tisser, conjonction de la verticalité de la chaîne et de l'horizontalité des attaches qui la lient à la maison, s'apparente aux conceptions locales du féminin et du masculin toujours en relation. Ensuite, à un second niveau, la présence de cette dualité est rappelée dans la technique de tissage, manière de faire comme représentation d'une manière d'être.

Ourdir, retour au récit

Chaque nouvel ourdissage renouvelle le métier à tisser $-\mathrm{y}$ compris physiquement. Il le crée même puisque d'un point de vue lexical le terme azta n'existe qu'une fois l'ourdissage commencé et le métier est azta quand la chaîne est verticale. L'expression n'durou el azta, désignant l'opération d'ourdissage, signifie précisément «faire passer les fils devant d'un piquet à l'autre ", qui correspond à la phase de création de la chaîne : trois piquets sont enfoncés dans le sol de la cour de la maison afin de dévider la pelote en allant d'un piquet à l'autre pour constituer les nappes de la chaîne. Cette opération cruciale est précédée d'un partage de nourriture.

21 Avant de commencer le travail, les femmes réunies pour l'ourdissage consomme de la bsissa et du thé. La bsissa doit être présente dans la maison de la tisseuse. On retrouve ce mets le premier jour des noces, lors de la fête des sept jours après la naissance d'un enfant et lors de la circoncision. 

vie sociale : la richesse, la fécondité, le plaisir (goût), la durée, la protection. C'est un aliment réputé fortifiant et consistant. Grillée au feu puis mouillée (huile, eau) après avoir été moulue, la bsissa réunit des contraires : la succession du feu et de l'humide est, en termes de représentations, une association de l'intérieur et de l'extérieur qui explique pourquoi la bsissa est rattachée aux rituels précités. En effet, les vertus de la bsissa sont extrêmement positives car elle est la résultante de cette association idéale valorisée par les Douiri qu'est la relation intérieur/extérieur. Le feu et les aliments grillés sont toujours liés à l'extérieur : on les retrouve sous plusieurs formes, comme le foyer des cuisines à l'extérieur des maisons dans le vieux village, les viandes grillées toujours préparées et consommées à l'extérieur, les feux de palmes et de pailles dans les jardins, les feux rituels de la fête d'Achur' ${ }^{18}$. L'huile et l'eau sont associées à l'intérieur; ainsi, les viandes bouillies sont toujours préparées et consommées à l'intérieur, le pressoir à olive est situé dans l'équivalent de maisons creusées, l'eau a des qualités intrinsèques dont on se sert pour protéger un métier à tisser, un tissu juste terminé... partage de bsissa pendant cet ourdissage est un acte rituel qui va de soi, il n'est pas expliqué et n'est pas remis en cause: "La bsissa, lors du montage du métier c'est comme ça, la tradition, il faut toujours la bsissa». Si l'analogie entre le montage d'un métier à tisser, le tissage et la bsissa n'est pas donnée par le discours vernaculaire, le récit, par la relation qu'il établit, fournit l'explication de la commensalité préalable au geste technique, qui lie le tissage à la bsissa. Il se dégage de l'analyse une proximité, en termes de représentations, entre tisser et distribuer la nourriture rituelle. Par les analogies des commencements de l'ourdissage et des noces, cette légende met clairement en relation l'institution et les rituels de mariage avec le tissage, au sein du terroir de Douiret.

Sur le plan formel, le récit est ordonné. En premier lieu, le décor est posé par le questionnement de la femme à propos de l'avancée extrêmement rapide du tissage de ses tapis. Elle découvre ensuite, par le biais de la tamzat, le moyen de "tisser à deux ». Elle transmet à la société cette découverte par l'intermédiaire de son fils. La seconde partie de l'histoire fait allusion à la distribution de bsissa dont les restes se transforment en or après une nuit, tout comme pour les rituels de mariage où l'or des bijoux répond à la bsissa inaugurale des noces. Dans le récit, le tissage précède la bsissa et l'or, alors qu'à l'occasion des noces il intervient entre les deux. Cette chronologie hiérarchise la société de Douiret en soulignant ce qui englobe l'ensemble: le tissage transcrit concrètement l'être relationnel qu'est toute personne adulte à Douiret; il est présenté comme la valeur qui englobe et organise le rituel et l'institution du mariage.

Pour leurs critiques et suggestions sur ce texte, je remercie F. Grognet, A. Montigny et F. Cousin (MNHN) ainsi que les participants au séminaire Graad-EHESS. 


\section{BIBLIOGRAPHIE}

Bonte, Pierre

1999. «Travail, techniques et valeur. Contributions (nouvelles) au débat sur rites et techniques », pp. 15-50, in J-L. Jamard, A. Montigny et

F-R. Picon (eds), Dans le sillage des techniques, Hommage à Robert Cresswell. Paris : L'Harmattan.

Cresswell, Robert

2001. «L'apport de la technologie culturelle à l'étude des sociétés du Proche-Orient et du Maghreb », pp. 595-617, in D. Albera, A. Blok et C. Bromberger (eds), L'Anthropologie de la Méditerrané. Paris : Maisonneuve et Larose/MMSH.

Lacoste-Dujardin, Camille

1986. « Ogresse berbère et ogresse corse : images de la femme méditerranéenne ? », pp. 277-287, in Atti del Congreso Internazionale di Amalfi, 5-8 décembre 1983. Naples : Istituto Universitério Orientale.

Lefebure, Claude

1978. " Linguistique et technologie culturelle, l'exemple du métier à tisser vertical berbère », Techniques \& culture $3: 84-148$.

Levinas, Emmanuel

1983. «De l'un à l'Autre », Archivio di Filosofia 1-3.

1990 « De l'être à l'autre », in F. Lenoir (ed.), Le Temps de la responsabilité, entretiens sur l'éthique? Paris : Fayard.

Pardo, Véronique

2003. Tisser les relations sociales. Dans les rites et la matière, représentations de l'ordre social, des valeurs et de l'appartenance à Douiret, village berbérophone du sud-est tunisien. Thèse de doctorat en Anthropologie sociale, Université de Provence/Maison méditerranéenne des Sciences de l'Homme.

Warnier, Jean-Pierre

1999. Construire la culture matérielle. L'homme qui pensait avec ses doigts. Paris: Presses Universitaires de France.

\section{NOTES}

1. Dans ce village de montagnes, adossé aux contreforts du jebel abhiod, la population parle deux langues : une variante locale du berbère nommée chelha et l'arabe dialectal tunisien émaillé des particularismes régionaux.

2. Concept d'entre-nous emprunté à Emmanuel Lévinas $(1983,1990)$. Le « nous » est la société, la totalité; l'entre-nous, à Douiret, correspond à l'ensemble des relations qui créent les Douiri et sont créées par eux L'approche de l'être relationnel chez Lévinas correspond à la conception locale de la personne (faite de deux) et non semblable à notre individu moderne.

3. Les données ethnographiques de cet article ont été recueillies dans le village de Douiret lors d'une mission effectuée de janvier 1998 à février 1999, puis lors de 
plusieurs autres séjours entre juillet/août 1999 et septembre 2001. Ces recherches ont fait l'objet d'une thèse de doctorat (Pardo 2003).

4. Les tapis de laine nommés tazarbit en chelha ou kilim en arabe sont constitués de bandes de couleur alternées, chaque bande étant monochrome; les couvertures tissées, zaouret en chelha et farachiya en arabe, sont en général bicolores (laine vierge et une laine teinte). Les tapis et les couvertures les plus valorisés sont ornés de motifs géométriques organisés selon différentes règles de symétrie; le terme margoum est alors ajouté à leur nomination.

5. Les femmes tissent encore le tissu servant à confectionner les vêtements couvrants d'hiver (burnous et cachabiyya) pour les hommes. Les voiles féminins étaient, quant à eux, les pièces les plus prestigieuses anciennement tissées à Douiret, car ils réalisaient l'association de la maîtrise parfaite de la technique du tissage et la connaissance des canons de la société en termes de couleurs, matières, symétries... Ces voiles, ceux nommés tabahnougt/bahnoug ainsi que ceux désignés par le terme tajir'a, sont toujours portés, valorisés et préservés.

6. Les Douiri utilisent le terme kilim pour désigner « dans le dialecte arabe régional » les tapis tissés (et non les tapis noués de Jerba ou Kairouan). Il s'agit du nom générique d'origine turque désignant une technique particulière de tissage. Dans le village, kilim correspond aux tapis tissés présentant en alternance des bandes polychromes de largeur identique, horizontales et parallèles aux ensouples. Quand nous parlons de kilim, il s'agit, sauf précision, du sens donné localement à ce terme et non celui du vocabulaire général et international du tissage.

7. Sur le plan littéraire, ce récit peut sembler construit de deux parties distinctes artificiellement réunies pour l'analyse. Il n'en est rien; il a été relevé ainsi et le lien existe du fait de l'énonciation, du ton et de l'emphase du récitant, même s'il n'est marqué par aucun mot de liaison.

8. Nous traduisons par « terroir » le terme chelha de «tamourte », lequel désigne à la fois le village et la terre qui appartient à un groupe donné, terre cultivable mais également terre des ancêtres. La polysémie du terme «tamourte » rencontre celle du terme français terroir dont l'une des occurrences (Le Robert) correspond à une terre censée porter des habitants qui l'influencent et sont influencés par elle.

9. Si la personne exprime ce terme en arabe dialectal, elle dira ghoula, souvent traduit dans les contes par le mot français « ogresse ». Nous n'utilisons pas cette traduction car ce terme est connoté en français d'un sens très différent de celui conféré à la tamzat dans la région concernée. D'après le dictionnaire Le Robert, « une ogresse est une géante se nourrissant de chair humaine »; rien de tel dans le portrait de la tamzat berbère des montagnes du sud-est tunisien. Ou alors il serait possible de prendre « ogresse » au sens de Camille Lacoste-Dujardin (1986) et d'en faire une métaphore bien singulière de la femme méditerranéenne.

10. L'offrande de bsissa lors des visites dans les sanctuaires est courante dans d'autres régions de Tunisie, à Jerba notamment, mais jamais rencontrée à Douiret où l'on va plutôt cuire et partager de la nourriture sur place : beignets, pain, couscous... Il est toutefois fait allusion ici à un type d'offrande courant, qui consiste à laisser à un endroit un peu de nourriture ou d'eau censées protéger l'offrant.

11. La cuisson sur un feu dans la cour est exceptionnelle et n'intervient qu'en deux occasions : la préparation de la bsissa et la cuisson du plat d'Achur'a.

12. Aujourd'hui, on amène ces ingrédients grillés à la ville de Tataouine pour les faire moudre; ils étaient autrefois moulus sur place avec un moulin manuel. 
13. Émile Laoust (1912) rattache la racine zett (tisser) au mot tazettat qui, chez les Berbères du Maroc, désigne la protection accordée à tout individu voyageant en territoire étranger ou la rétribution reçue par le protecteur en échange de sa protection. Le mot est à mettre en relation avec le verbe $z d$, «tisser » mais aussi «tresser, tricoter » (Lefébure $1978: 89$ ).

14. La tamzat a par nature la possibilité de se rendre visible ou invisible.

15. L'être le plus extérieur est féminin, la transmission de la technique passe par le fils. 16. Cette analyse fait l'objet de ma thèse de doctorat où j'ai montré qu'il était possible de dégager les figures de l'appartenance au groupe au travers d'un faisceau de faits techniques, esthétiques et rituels. L'étude lexicale des termes chelha a permis, dans le cadre de la description du métier à tisser et du tissage, de comprendre certains des enjeux d'appartenance à cette société. Plus encore, la définition de l'esthétique au travers d'un style douiri a permis de dégager la logique des mécanismes de cette appartenance à une société locale particulière.

17. Tant qu'ils ne sont pas mariés (même très âgés), un garçon, afrurt, et une fille, tafrurt, portent ces noms qui qualifient l'enfant. À la suite des noces, le garçon devient ariez, soit homme, adulte (homme marié), et la fille, femme tamatout (mariée, celle qui enfante, qui allaite - le complément de sens « qui allaite » n'est pas une donnée issue de mes recherches mais du séminaire de Cl. Lefébure, EHESS 2002-2003).

18. Autrefois, lors de la fête d'Achur'a, les enfants et surtout les garçons, encouragés par les chants de leurs grands-mères, devaient sauter au-dessus de grands feux en évitant de se brûler afin de montrer leur courage et leur droiture.

INDEX

Mots-clés : femmes, tissage, Tunisie, Douiret, savoir-faire

\section{AUTEUR}

\section{VÉRONIQUE PARDO}

UMR 5196 « Techniques et culture », CNRS, 27 rue Paul Bert, 94204 Ivry-sur-Seine Cedex; Ater, Université Marc Bloch de Strasbourg 